\section{Fracture du capitulum}

\section{Fracture of the Capitulum}

\section{H. Ait Benali • M.S. Berrada • M. El Yaacoubi}

Reçu le 29 juin 2014; accepté le 11 septembre 2014

(C) SFMU et Lavoisier SAS 2014

Une femme de 34 ans sans antécédent est amenée aux urgences chirurgicales pour un traumatisme du coude droit suite à un accident domestique. Il s'agit d'une chute sur la paume de la main, coude en extension. L'examen clinique retrouve une douleur et une impotence fonctionnelle totale du coude associées à un œdème important. Il n'y a pas de déformation ni de signe de compression nerveuse ou vasculaire. La radiographie de face est strictement normale. Le diagnostic est posé sur la radiographie de profil du coude qui montre un fragment osseux détaché en forme de demi-lune en regard de la palette humérale. Ce fragment correspond au capitulum ou condyle latéral qui normalement s'articule avec la tête radiale en absence de fracture. La patiente a bénéficié d'un traitement chirurgical par vissage direct en compression suivi d'une immobilisation plâtrée pendant trois semaines et d'une rééducation fonctionnelle. L'évolution a été très favorable, marquée cliniquement par une indolence complète et une mobilité correcte avec une flexion-extension complète. La fracture du capitulum est une fracture-séparation articulaire frontale et partielle de la palette humérale. Il s'agit d'une fracture rare, elle représente moins de $1 \%$ de l'ensemble des fractures du coude. Elle pose des problèmes diagnostiques en raison de l'absence de déformation du coude et la discrétion des signes radiologiques. Elle est surtout visible sur l'incidence de profil. L'image en demi-lune ou double contour est caractéristique de ce type de fracture. La tomodensitométrie avec reconstruction tridimensionnelle est l'examen le plus performant pour faire le diagnostic avec précision. Le traitement est essentiellement chirurgical. La

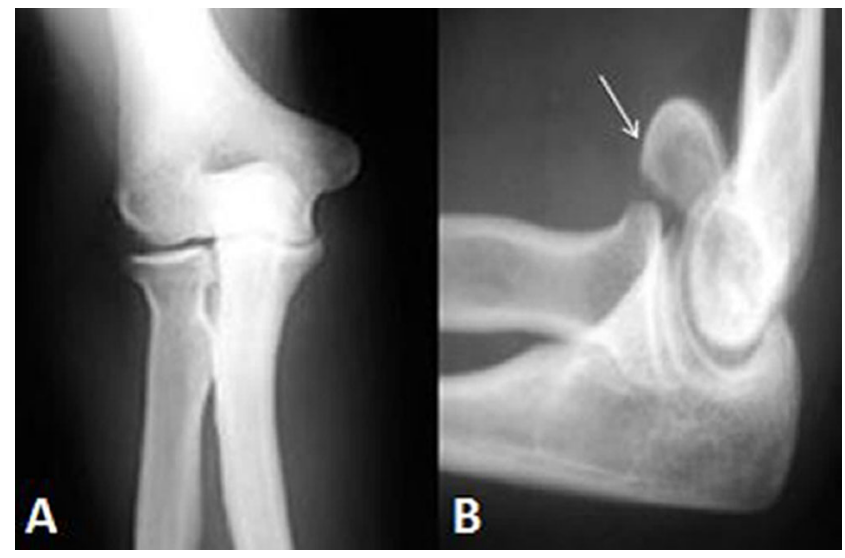

Fig. 1 Radiographie du coude de face (A) ne montrant pas de signes de fractures et de profil (B) montrant le détachement d'un fragment osseux correspondant au capitellum (flèche)

rééducation postopératoire est indispensable pour obtenir des résultats fonctionnels satisfaisants [1].

Liens d'intérêts : H. Ait Benali, M.S. Berrada et M. El Yaacoubi déclarent ne pas avoir de lien d'intérêt.

\section{Référence}

1. Hachimi K, Hattouma N, Sennoune B, et al (2004). Le traitement chirurgical des fractures du capitellum chez l'adulte (à propos de huit cas). Chir Main 23:79-84

\author{
H. Ait Benali $(\bowtie) \cdot$ M.S. Berrada $\cdot$ M. El Yaacoubi \\ Université Mohamed V Souissi, \\ service de traumatologie-orthopédie, hôpital Avicenne, \\ CHU Rabat, Maroc \\ e-mail : abhicham@hotmail.fr
}

Service de traumatologie-orthopédie, hôpital Avicenne, CHU Rabat 